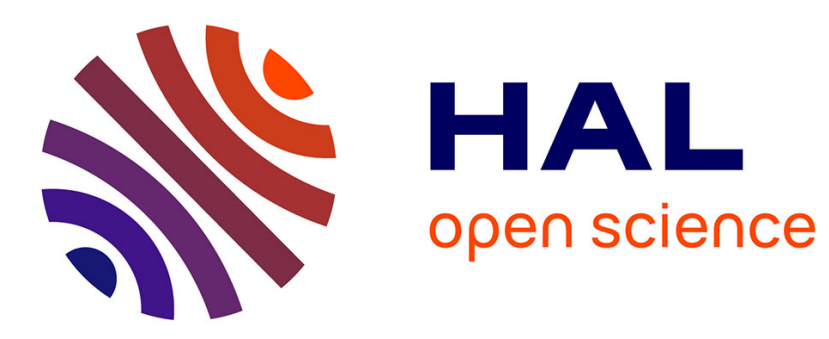

\title{
Reaction-Diffusion Network For Geometric Multiscale High Speed Image Processing
}

Stéphane Binczak, Tadeus Sliwa, Sabir Jacquir, Jean-Marie Bilbault

\section{To cite this version:}

Stéphane Binczak, Tadeus Sliwa, Sabir Jacquir, Jean-Marie Bilbault. Reaction-Diffusion Network For Geometric Multiscale High Speed Image Processing. Image and Vision Computing, 2010, 28, pp.914-926. 10.1016/j.imavis.2009.11.008 . hal-00583468

\section{HAL Id: hal-00583468 \\ https://u-bourgogne.hal.science/hal-00583468}

Submitted on 5 Apr 2011

HAL is a multi-disciplinary open access archive for the deposit and dissemination of scientific research documents, whether they are published or not. The documents may come from teaching and research institutions in France or abroad, or from public or private research centers.
L'archive ouverte pluridisciplinaire HAL, est destinée au dépôt et à la diffusion de documents scientifiques de niveau recherche, publiés ou non, émanant des établissements d'enseignement et de recherche français ou étrangers, des laboratoires publics ou privés. 


\title{
Reaction-Diffusion Network For Geometric Multiscale High Speed Image Processing
}

\author{
S. Binczak ${ }^{\text {a,*,1 }}$, T. Sliwa ${ }^{\text {b }}$, S. Jacquir ${ }^{\text {a }}$, J.M. Bilbault ${ }^{\text {a }}$ \\ ${ }^{a}$ LE2I CNRS UMR 5158, Aile des Sciences de l'Ingénieur, Université de \\ Bourgogne, BP 47870, 21078 Dijon Cedex, France \\ ${ }^{\mathrm{b}}$ LE2I CNRS UMR 5158, Université de Bourgogne, Route des plaines de l'Yonne, \\ BP 16, 89010 Auxerre, France
}

\begin{abstract}
In the framework of heavy mid-level processing for high speed imaging, a nonlinear bi-dimensional network is proposed, allowing the implementation of active curve algorithms. Usually this efficient type of algorithm is prohibitive for real-time image processing due to its calculus charge and the inadequate structure for the use of serial or parallel architectures. Another kind of implementation philosophy is proposed here, by considering the active curve generated by a propagation phenomenon inspired from biological modeling. A programmable nonlinear reaction-diffusion system is proposed under front control and technological constraints. Geometric multiscale processing is presented and a discussion about electronic implementation is opened.
\end{abstract}

Key words: Image analysis, Multiscale geometry, Nonlinear signal processing PACS: 87.57.Nk, 07.05.Pj, 05.45.a 


\section{Introduction}

Background framework of this work consists in the development of processing systems and their electronic implementation for high speed image intelligent sensors. Nowadays, many image processing techniques, particularly most of the linear or probabilistic ones, are included in real-time systems, but many families of mid-level techniques still remain unusable for such time processing requirements.

For high speed imaging applications, only some families of simple low-level techniques can be used, other techniques being applied on recorded data. For high speed imaging treatments, the approach of artificial retinas consisting in locating processing at the pixel stage gives some good results [1-3]. However, an important disadvantage lies with this approach: Many treatments either remain very difficult to adapt to retinas architectures or imply development of specific architectures. To avoid this disadvantage, a generic way of image processing is proposed.

Mathematical morphology [4,5] has been specifically developed to meet image processing expectations. Therefore, it leads to solve a major part of image processing problems and is widely used. High speed electronic implementations of basic morphological operators as dilations have already been studied, for instance with the use of a nonlinear electronic network [6]. However, the most important families of mathematical morphology treatments are based on two other main heavy operators which are geodesic reconstruction and watershed algorithm.

* Corresponding author.

Email address: stbinc@u-bourgogne.fr (S. Binczak).

1 Tel: +33 380396848 ; Fax: +33 380395910 
Different architectures have been built in order to implement these mid-level treatments [7-9], most of them using numerical technologies, able to do realtime image processing for usual speeds, but with a technological gap preventing high speed imaging applications.

In this context, a possible generalization of the geodesic dilation is explored by constraining its construction with compatibility regarding a second connexity notion linked to the regularity of the shape of the objects. It is not expressed algebraically, but through a more intuitive approach. In fact, geodesic dilation can be interpreted as a topological wave propagation starting from a marker whose evolution is defined by the structure of the image. PDE (Partial Differential Equations) are a very convenient tool to generate this propagation where features of the image are included as intrinsic parameters of the equations. Furthermore, some of the reaction-diffusion PDE include a notion of regularity for the propagation phenomenon. For some of them, it is even possible to adjust parameters defining the capacity of the wave to travel through straight zones, holes, breaks and so on. Therefore, it defines a kind of geodesicactive curve or region.

Another aspect is very important for the notion of objects' regularity: The scale from which the object is observed. But, carrying out multiscale analysis through successive multiple wave propagation is contrary to the high speed imaging technological gap. In order to reduce treatment times, the ideal case would be to achieve a multiscale analysis with only a one-pass wave propagation. In fact, a phenomenon producing propagation of wavefronts with increasing regularity along the height of the transition part is studied.

Besides, a systematic way of exploring dynamical systems described by PDE has been obtained by developing electronic circuits, which were very efficient for real-time analysis. These techniques led to analog computers, electrical 
lattices [10] or to the more general concept of Cellular Neural Network [11] $(\mathrm{CNN})$. Although power computing has experienced an exponential growth, these dedicated systems have benefited from integrated electronics and still remain an alternative for high speed processing. Thus, it is possible to use this kind of electronic device to obtain propagation speeds allowing it to overcome the high speed imaging technological gap.

Therefore, the purpose of this study is to propose a reaction-diffusion system with interesting properties, defined under technological constraints in order to suggest an electronic implementation. This article is organized as follows: A choice of reaction-diffusion system is presented in section 2; then, the process of propagation of topological waves is studied according to the distribution of the local diffusive parameters. This provides a way to control the propagation paths of the travelling waves and to deduce a generic image processing method. In section 3, this image processing is applied on specific images, enabling edge restoration/ interpolation or one-pass multiscale approximation. This section is concluded by a preliminary result on grey level based segmentation, indicating that this system can lead to generic processing. Section 4 is devoted to open discussion on some possible implementation of an electronic circuit which could be able to perform high speed image processing. Finally, section 5 concludes and gives perspectives of this study.

\section{A discrete bistable system as a Multiscale Regularity PDE}

Not all interesting reaction-diffusion PDE have realistic electronic implementation. When dealing with regular lattice, the simplest way to connect nodes leads to the classical heat equation. Unfortunately, in this medium, no stable 
propagation waves can exist. In order to overpass this limitation, a possible modification is to use the most general reaction-diffusion equation :

$$
\frac{d v_{n, m}}{d t}=D_{n, m}\left[v_{n-1, m}+v_{n+1, m}+v_{n, m-1}+v_{n, m+1}-4 v_{n, m}\right]-f\left(v_{n, m}\right) .
$$

From a dynamical system analysis, the minimum number of equilibrium points is three in order to assure the existence of stable propagating front wave with controllable shape. This leads naturally to the bistable equation.

In this section a study of the propagation in a system modeled by this equation is presented. It will be shown that, although this model is quite simple and its analog electronic implementation easily reachable, it allows the paths of propagating waves to be controlled, and therefore leads to deduce some interesting properties for image processing.

Let us consider a bi-dimensional regular discrete $(N, M)$ - length grid $\Omega$ on which the following bistable diffusive system is defined:

$$
\begin{aligned}
& \frac{d v_{n, m}}{d t}=D_{n, m}\left[v_{n-1, m}+v_{n+1, m}+v_{n, m-1}+v_{n, m+1}-\right. \\
& \left.4 v_{n, m}\right]-v_{n, m}\left(a-v_{n, m}\right)\left(1-v_{n, m}\right)
\end{aligned}
$$

where $D_{n, m}$ is a local diffusion parameter, $a$ a threshold parameter and $v_{n, m}$ corresponds to the information located at node $(n, m)$ (for instance, the intensity value of the pixel $(n, m))$.

The system is completed by the Neumann conditions (zero-flux conditions) on the border $\partial \Omega$ of the definition domain $\Omega$, so that

$$
\frac{\partial v_{n, m}}{\partial \eta}=0 \quad \text { if }(n, m) \in \partial \Omega
$$


where $\frac{\partial}{\partial \eta}$ denotes the outer normale derivative at the boundary.

In the following section, propagation phenomena emerging from this system are investigated, from which a generic image processing method is then deduced.

\subsection{Wavefronts in the homogeneous grid case}

For the sake of simplicity, we first consider the case where the local diffusion parameter is a constant so that

$$
D_{n, m}=D \quad \forall(n, m) \in \Omega
$$

This particular system corresponds to a discrete version of the FitzHughNagumo PDE (without recovery term) which was established to describe propagation phenomena in various biological systems. In a one-dimensional space, it allows the study of the electric propagation of the leading edge of an action potential, the neuronal information travelling along the membrane of the nerve fibers $[12,13]$. Its discrete form (discrete Laplacian) corresponds to the myelinated nerve fibers case. In a continuous bi-dimensional space, it is widely used to study wavefront propagation in myocardial tissues [14].

In the uncoupled case, i.e. when $D=0, v_{n, m}=0$ and $v_{n, m}=1, \forall\{n, m\}$ are two attracting steady states, while $v_{n, m}=a, \forall\{n, m\}$ is an unstable equilibrium point of the system, acting as a threshold.

In case of strong coupling, i.e. when $D$ is large, we expect that a travelling wave will propagate depending on the value of $a$ with a constant speed so that if $a<1 / 2(a>1 / 2$ resp.), the steady state $v=1$ ( $v=0$ resp.) will propagate at the expense of the steady state $v=0(v=1$ resp.). When $a=1 / 2$, no 
propagation occurs [15].

The response of the system, especially the shape of a wavefront, depends not only on the parameters of the system, but also on the initial conditions.

Let a marker be defined as a particular family of initial conditions for which $v_{i, j}(t=0)=1$ for a set of $(i, j)$ values, $v_{k, l}(t=0)=0$ otherwise. The symmetry of the marker will determine the kind of travelling waves and its spatial shape. Among them, the two main propagating structures are the planar and the circular wavefronts. The following results are illustrated using numerical simulations obtained using a $4^{\text {th }}$ order Runge-Kutta algorithm.

\subsubsection{Planar waves}

They emerge from a rectangular marker, as illustrated in Fig. 1 and 2 where two planar wavefronts propagate in opposing directions.

The symmetry induced by this choice of marker allows us to reduce the system to a one-dimensional space problem, expressed by:

$$
\frac{d v_{n}}{d t}=D\left[v_{n-1}+v_{n+1}-2 v_{n}\right]-v_{n}\left(v_{n}-a\right)\left(v_{n}-1\right)
$$

This system of equation has been widely investigated to study the neural propagation in myelinated nerve fibers. From these studies, some important results can allow us to characterize the process of propagation, although no explicit overall analytical expression of the wavefront is available.

In the first place, when the coupling between nodes is strong, the differentialdifference system (5) can be written, using a continuum approximation, so that

$$
\frac{\partial v}{\partial t}=D \Delta v-v(v-a)(v-1)
$$


where $\Delta$ denotes the Laplacian operator. Using a travelling-wave analysis [15], one can express the propagation wave profile according to the travelling coordinate $\xi=n-u t$ and initial conditions $\xi_{0}$, and the unique velocity $u$ of this topological wave as a function of $D$ and $a$, so that

$$
\begin{aligned}
& v(\xi)=\frac{1}{2}\left[1 \mp \tanh \left[\frac{\xi-\xi_{0}}{\sqrt{8 a D}}\right]\right] \\
& u= \pm(1-2 a) \sqrt{\frac{D}{2}} .
\end{aligned}
$$

Eq. (7) indicates that the width of the wavefront decreases when $D$ becomes smaller (this relationship remains valid even in a discrete system), while eq. (8) tells us how the parameter $a$ controls the propagation, depending on its value in comparison with $1 / 2$. In particular, it shows that the velocity increases when $a$ decreases. Note also that the \pm sign in eq. (8) corresponds to a bidirectional propagation. Qualitatively, these observations remain valid in the discrete case. For instance, Fig. 2 illustrates bidirectional wavefronts whose velocity is constant.

Nevertheless, a major feature due to discreteness is the failure of the propagation when $D$ is smaller than a critical non-zero value $D^{*}>0$, which is missed in the continuum approximation. In this case, the wavefront is pinned [15-17], as illustrated in Fig. 4. From [17], an asymptotic expression of this parameter when $a \longrightarrow 0$, is

$$
D^{*}=\frac{1}{4} a^{2}
$$

which is in accordance with the results presented (In this case $D^{*}=0.0025$ ). 


\subsubsection{Circular wave}

This kind of travelling wave emerges when the marker tends to be circular, or has a circular symmetry, as illustrated in Fig. 3.

The spatial shape determines the velocity of the wavefronts, so that, in a continuous system [18], a convex travelling wave propagates slower that a planar one. This behaviour matches the comparative results in Fig. 4 when the diffusive parameter $D$ becomes large enough.

As $D$ decreases, the wavefront width becomes smaller, emphazing the discreteness of the system. Therefore, a circular distribution along discrete nodes is no longer possible, and is transformed into a combination of planar waves with different wave vectors. As a consequence, "circular wave" and planar wave velocities eventually merge as $D$ decreases. In addition, propagation fails for the same value $D^{*}$ of $D$. From now on, we will assume that $D^{*}$ is not a function of the kind of travelling waves, but only determined by the threshold parameter $a$.

\subsection{Wavefronts in an inhomogeneous grid case}

Dramatic in biological systems, the propagation failure phenomenon can be useful to develop new approaches in image processing. Contrary to classic cases, the point of interest is indeed the blocking case, allowing the path of the topological travelling wave to be controlled and leading to mid-level image processing. An image is then defined as a discrete bi-dimensional grid where nodes correspond to pixel sites. Each node is coupled to its nearest neighbours in a diffusive manner weighted by the local intrinsic information of the image. The main idea is to initiate a wavefront and let it propagate until it reaches 
an object $\Theta$ to be detected. In order to prevent further propagation, we now impose the following rule in eq. (2):

$$
D_{n, m}=\left\{\begin{array}{lc}
D_{p}>D^{*} & \text { if }(n, m) \notin \Theta \\
0 & \text { othervise }
\end{array}\right.
$$

As $D_{p}>D^{*}$, propagation is possible if an appropriate marker is set outside the object. In a complementary way, $D=0$ within the object, pins this wavefront at its borders, as illustrated in Fig. 5: The object is composed of two horizontal strands whose nodes are uncoupled. A rectangular marker is set at the top lines of the grid initiating a planar wave propagating along the vertical axis. Due to the propagation failure criterium, this wave stops at the border of $\Theta$ and can only extend through the corridor separating the two parts of the object. Because of this symmetry breaking, a circular wave emerges and propagates in the bottom part of the grid. Eventually, a stationary state is obtained so that the whole object is surrounded by the final state $v=1$.

In addition, other new features appear from this inhomogeneous coupling. As presented in Fig. 6, a wavefront can not only be blocked due to the parameters of the system, but also to its geometry.

Compared to the case of Fig. 5, the wavefront is pinned in the corridor, leading to another final state, because of a larger diffusive parameter $D_{p}$. The corresponding mathematical mechanism leading to this blockage is based on the existence of a stationary wave due to a maximum curvature prohibiting the emergence of a travelling wave. To put it simply, a larger width of the wave implies that the wave cannot propagate through narrow paths. Note that this result can also be observed in a bidimensional continuum space $[18,19]$. A systematic study of the relationship between the diffusive parameter $D$, the 
width $W$ and the thickness $T$ of the corridor is presented in Fig. 7. The results indicate that there exists a critical value $D_{m}$ above which no propagation can emerge from the corridor. Obviously, the larger and thinner the corridor is, the wider a propagating wave can be, therefore, $D_{m}$ increases. As discussed in the following section, this property can be interesting to integrate objects or to delevop active curves.

In all the previous cases, it was implied that a marker necessarily generates a travelling wave. Actually, specific initial conditions are to be taken into account depending on the parameters of the system [20]. More precisely, there exists a threshold surface for which the nodes must be above the excitation threshold $a$ to ensure the initiation of a fully developed travelling wave. If an initial condition does not satisfy this requirement, the initial surface being smaller than the threshold one, then it decays to zero. Let us restrict this problem to initial conditions corresponding to a square marker of side $W$, as defined previously.

The results are presented in Fig. 8, showing that the size of the marker is a function of the diffusive parameter $D$. As the system becomes more diffusive, the marker is more spread on the grid, implying that fewer nodes will be above the excitation threshold. The purpose of our study is to initiate a propagation from a marker of whatever size. Therefore, we impose these initial conditions as Dirichlet ones, by setting the following Marker-rule:

$$
D_{n, m}=0 \quad \text { if }(n, m) \in \text { Marker }
$$

It corresponds to a forced propagation condition, implying the always successful initiation of a propagating wave [17], if the local diffusive parameter $D$ in the neighbourhood of the marker is above propagation failure criterium $D^{*}$. 


\subsection{A Generic Image Processing Method}

Now let us build a generic image processing method. For this, we will use the marker principles of geodesic mathematical morphology with the possibility of combining them with other image processing techniques.

$A$ and $B$ will denote sets or, with the same notation, indicate set functions, but they are more generally intended to be greyscale image quantities as different transforms of intensities, including duality to allow most possible types of operators, of the norms of local vectors of directional derivatives, or even of scalar-type results of other image processing as for example linear filtering. $A$ and $B$ can be determined from the same image or from different images. Here $A$ will represent binary markers from which the propagation phenomenon starts, and $B$ the topological constraint derived from the image. The couple 
of scalar discrete functions $(A, B)$ defines the following equation:

$$
E_{\varepsilon}(A, B)=\left\{\begin{array}{l}
\frac{d v_{n, m}}{d t}=\frac{D(B)}{\varepsilon}\left[v_{n-1, m}+v_{n+1, m}+\right. \\
\left.v_{n, m-1}+v_{n, m+1}-4 v_{n, m}\right]-f_{a}\left(v_{n, m}\right) \\
f_{a}(v)=v(a-v)(1-v) \text { with } a<\frac{1}{2} \\
D(B)=\frac{1+\tanh (20 B-12)}{2} \\
\left.v\right|_{t=0}=A
\end{array}\right.
$$

Here $a$ will have the value 0.1 and the definition domain $\Omega$ will be the $(n, m)-$ length grid.

The system is completed by the Neumann conditions (zero-flux condtions) on the border $\partial \Omega$ of the definition domain $\Omega$.

The Marker-rule expressed by eq. (11) is supposed set, otherwise specified. This choice of constructing $D(B)$ corresponds to a bimodal distribution of the local diffusive parameter separated by $D^{*}$, allowing the control of the propagating paths.

The propagation phenomenon defined by $E$ tends to a convergence state noted $v_{\varepsilon}^{\infty}(A, B)$, theoretically corresponding to infinite $t$ but practically a millisecond value will be sufficient in most cases. $v_{\varepsilon}^{\infty}(., B)$ is idempotent if eq (11) is set 
or not, in the mean of

$$
v_{\varepsilon}^{\infty}(A, B)=v_{\varepsilon}^{\infty}\left(v_{\varepsilon}^{\infty}(A, B), B\right) .
$$

Depending on $\varepsilon$, several properties arise:

- $v_{\varepsilon}^{\infty}(., B)$ tends to identity, when $\varepsilon \longrightarrow+\infty$,

- $v_{\varepsilon}^{\infty}(A, B) \longrightarrow 1_{\Omega}$, when $\varepsilon<\min \left(D(B), D_{m}\right)$, if the marker rule is set,

- $v_{\varepsilon}^{\infty}(A, B) \longrightarrow 1_{\oslash}$, when $\varepsilon<\min \left(D(B), D_{m}\right)$, if the marker rule is not set.

Then, let us define the main image processing operator as:

$$
\Phi_{\varepsilon, h}(A, B)=\left\{v_{\varepsilon}^{\infty}(A, B) \geq h\right\} .
$$

The propagation phenomenon is similar to the geodesic propagation, but with an additive scale of regularity constraint, defined by the couple variables $(\varepsilon, h)$ with $\varepsilon$ defining the magnitude order of the scale of regularity of the one-pass propagation and $h$ a thresholding along the scale regularity consequently to the choice of $\varepsilon$ in the final result when the propagation is definitively blocked by the topological constraint. It is equivalent to fix $\varepsilon$ and deduce $h$ or to fix $h$ and deduce $\varepsilon$. Eq. (14) leads to the immediate following properties:

- When $\varepsilon \longrightarrow 1^{+} / D^{*}, \Phi_{\varepsilon, h}(A, B)$ approaches the geodesic reconstruction of $B$ marked by $A$.

- $\Phi_{\varepsilon, h}(., B)$ increases with fixed regularity scale parameters and increases with its two regularity scale parameters increasing independently.

- $\Phi_{\varepsilon, h}(A,$.$) decreases with fixed regularity scale parameters and decreases$ with its two regularity scale parameters increasing independently. 
It allows the generation of many morphological-type analysis techniques, constructed as classic techniques through the morphological theory, but with some advantages from the point of view of active curves (considering the evolving front) or regions (considering the interior of the evolving curve) as their greater regularity, without the topological problems of the deformable templates. Warning: if the Marker-rule is not imposed, there is no strict extensivity property but the authors think that a low extensivity property depending on the observation scale could be defined, extending algebraic closing, opening, granulometries, convexity spectra and so on.

As illustrated by the numerical study, $\varepsilon$ plays the role of a main geometric scale parameter and $h$ a secondary geometric scale parameter. The $>$ sign produces an active geodesic region approach, $<$ producing a dual region approach, whilst replacing it by $=$ produces an active geodesic curve approach. Other operators can be derived from the extrema of the $\Phi_{\varepsilon, h}(A, B)$ function. For the following section, $c$ represents $1 / \varepsilon$.

\section{Numerical Study}

In this section, we characterize the propagation of waves in more complex and representative structures. The aim of this section is to extract from simple geometric cases the effects of topological and morphological structures on the propagation in order to illustrate (by using a $4^{\text {th }}$ order Runge-Kutta algorithm) its generic image processing possibilities. Among them, we focus on the ability of this system to reveal some multi-scale geometric information. Finally, we show that segmentation processing can also be reached. 


\subsection{Scale and propagation blockage}

First, we illustrate the effect of the scale factor on the propagation break. We turn to the geometrical aspect, so binary images are sufficient. In this case, $D(B)$ is nearly equal to $B$ which comes directly from the image. Figure 9 shows the sifter behaviour of the propagation: Its capacity to propagate through a corridor. For a given $c$, there exists a minimum corridor width above which waves can propagate though. The marker is the same in each case, but $c$ is not the same for each propagation: It is growing from left image to right image. It works as if we were looking at the corridor from a higher distance: It seems to become thinner and thinner. Then, we can see that the minimum corridor propagation width is growing with $c$.

For a given $c$, the Fig. 10 illustrates that the propagation blockage has an isotropic aspect, in the case where the size of the geometrical features of the image is sufficiently large compared to the size of the mesh of the system. Figure 11 illustrates the same behaviour but with a rather porosity effect: the more $c$ increases, the less propagation penetrates in the porous medium.

In a sense, propagation becomes more visquous. It could be interpreted as if each elementary part of the propagation front was blocked by a virtual dilation whose size is determined by the scale $c$. Therefore, $c$ can also be denoted as the "scale of observation". Note that it acts as if this scale determines a size for a class of discrete negligible details. It is also true for the marker, if the Marker-rule is not used: If its measure is under this minimal size, it is equivalent to the null region and the propagation falls before disappearing to the null function.

This viscosity aspect induces an immediate property of interpolation or restora- 
tion of the geometric borders, with $c$ determining the capacity of interiors/exteriors separation of the propagation phenomena, as illustrated in Fig. 12.

\subsection{One-pass multi-scale approximation}

Figure 13 shows that, when using sufficient $c$, the propagation acts like a viscous gauge, producing a viscous hull-like effect: The object is located between the two final states, allowing the definition of an area of interest in the image. Then, we can see in the following Fig. 14, with levelling the result of this one pass propagation, that we obtain multiscale geometric information: Lower levelling produces higher level of details and higher levelling produces a higher level of approximation.

If we push the levelling to its limits, we can almost obtain the initial shape of the object for very low levelling and sorts of approximation hulls for levelling near one. This scale aspect of h depends on the primary scale determined by $c$ : In fact, it is a secondary variation parameter around a sort of mean scale parameter. If $c$ is higher, it produces a panel of viscous-hulls.

If $c$ is smaller, it produces a panel of de-noised interiors/exteriors. A middle $c$ produces a sort of progressive elimination of breaks of the initial shape. The value of $c$ has to be adjusted depending on the application aim. An interpretation of means of measure of defects on manufactured objects on output of a production plant could be profitable: In a one go, it allows the quantification of noise, breaks and distortion/deformation, through a measure-function consisting in extracting one or more attributes for each $h$, giving a description of the shape. It can also be seen as a multiple pre-processing step between other treatments, leading to obtain more robust final results. With another 
kind of markers (grid-like ones for example), it could even be used for characterizing patterns. Moreover, identifying relationships between scale and length or surface of penetration (in the case of porous media for example), fractal spectrum-like descriptors could be obtained, leading to facilitate the distinction between rugosity and noise.

Note also that some fusion/separation aspects appear when $h$ varies, allowing the subdivision of geometric parts of objects, as if successively adding the effects of several centrum of propagation. Therefore, we can view the object as with a multiple geometric description, all this in one go. Indeed, there is an effect of front touch which could certainly be used for SKIZ-like based image processing algorithms. In all situations, it can imply artificial retinas allowing the storage of multiple scale geometric analysis in the level of the output of one stationary state from an electronic network based only on variable couplings. It is also possible to consider the multiscale geometrical analysis as a fuzzy segmentation function, as shown in Fig. 15. Here, the main scale parameter is high and the secondary scale parameter defines the connection between objects. As a first approach, we have a global group of objects, then divided into two groups with a large connection or not, and finally into four elementary objects. In fact we have multiple second connectivity distributed in a hierarchical structure due to its monotony regarding the level parameter, as for segmentation trees, but here stored in an image result. This can be considered as a fuzzy result and manipulated through fuzzy operators with other image results in order to obtain an analysis with multiple descriptions. This kind of technique could be used for sensor fusion information. 


\subsection{Grey level based segmentation}

In order to obtain grey level based segmentation, it is straightforward to include an estimation of the gradient information in the topological constraint derived from image, that is $B$. B has naturally to vary in a monotonic way with gradient. For simplicity, the choice of B has been realized by including a normalization on a power function of the gradient magnitude. Finally, with the following Fig. 16, we illustrate the result produced with :

$$
B=1-\frac{\left|g_{X}+i g_{Y}\right|^{1 / 4}}{\max _{\Omega}\left(\left|g_{X}+i g_{Y}\right|^{1 / 4}\right)},
$$

where $g_{X}$ is the numerical gradient of the image, corresponding to the differences in the row direction and $g_{Y}$ is the numerical gradient of the image, corresponding to the differences in the column direction.

The subsequent values $D_{n, m}$ are shown in the bottom right inset of Fig. 16. In this example, two markers are imposed, one in the background between the two columns, and the second one, inside the right cheek (see the top left inset). The blocked frontwaves are presented in the top right inset and in superimposition with the initial image in the bottom left inset. The results indicate that this choice of $B$ gives a very good greyscale segmentation for both areas. Note also that for a complete segmentation, we could, for instance, use a grid of markers. An important remark, when dealing with grey levels, is that the propagation depends not only on the density of the obstacle but also on its height, therefore the choice of $B$ may not be trivial. To conclude, the choice of $\mathrm{B}$ and the evaluation of corresponding segmentation performance is a major point which has to be systematically explored in a future work. 


\section{Electronic Implementation}

In order to reach high speed image processing, a specific programmable electronic device has to be conceived. However, it is not attended in this section to present an existing system but rather give a realistic scheme on how it could be realized. A possible electronic implementation for such tasks could be composed of a specific analog device controlled by a digital part. The major reasons are:

- to take advantage of the very fast signal processing character of analog circuits,

- to benefit from the fexibility of digital systems,

- to design it as a complement of classic numerical systems.

Figure 17 shows how the different parts of the treatment can be shared out. This mixed-signal architecture is split into three different parts: Two digital and one analog. The diffusive coupling $D_{n, m}$ is extracted depending on the choice of $B$ and the location of the marker(s). Because of multiple choices, this task is digital. The resulting values are then set in the analog network via DAC (Digital-to-Analog Converter).

The architecture of the analog network is presented in Fig. 18, details of which are shown in Fig. 19.

Initially, each capacity of each node is disconnected from the network, so that the markers can be introduced by loading the capacities (acting as analog memories). The local diffusive couplings $D_{n, m}$ are set by a multiplexing logic, by controlling the forward transconductance $g_{m}(n, m)$ of the OTAs [21] (Operational Transconductance Amplifier) of the coupling circuit via the associ- 
ated polarization currents. The initialization part is finished by simultaneously switching on the analog commutators via a synchronization control voltage $V_{\text {sync }}$ and resetting the digital counter. As the capacitors are now connected, using Kirchhoff's laws, the corresponding equations are

$$
\begin{aligned}
I_{n, m}= & g_{m}(n, m)\left[\left(V_{n-1, m}-V_{n, m}\right)+\left(V_{n+1, m}-V_{n, m}\right)+\right. \\
& \left.\left(V_{n, m-1}-V_{n, m}\right)+\left(V_{n, m+1}-V_{n, m}\right)\right] \\
I_{n, m}= & C \frac{d V_{n, m}}{d t}-I_{n l}\left(V_{n, m}\right),
\end{aligned}
$$

where $I_{n l}\left(V_{n, m}\right)$ is the current-voltage relationship of the nonlinear circuit. As the differential output voltage of the cascaded Gilbert cells [22] is connected to this input of an OTA, this current can be expressed so that, assuming that the devices are unsaturated,

$$
I_{n l}\left(V_{n, m}\right)=-g_{m} K^{2} V_{n, m}\left(V_{n, m}-a\right)\left(V_{n, m}-1\right),
$$

where $K$ is a multiplicative intrinsic constant of the Gilbert cell.

Note that this cubic function could be also realized by a less precise but more compact low voltage circuit as in [23]. From eqs (16) and (18), and after normalization, the system of equations modeling the network becomes

$$
\begin{aligned}
& \frac{d V_{n, m}}{d \tau}=D_{n, m}\left[V_{n-1, m}+V_{n+1, m}+V_{n, m-1}+V_{n, m+1}-\right. \\
& \left.4 V_{n, m}\right]-V_{n, m}\left(a-V_{n, m}\right)\left(1-V_{n, m}\right)
\end{aligned}
$$

with $D_{n, m}=\frac{g_{m}(n, m)}{g_{m} K^{2}}$ and the rescaled time $\tau=\frac{g_{m} K^{2}}{C}$.

The fact that $K \gg 1, g_{m} \gg 1$ and $C$, chosen so that $C \ll 1$ lead to obtain a very fast image processing network $(\tau \gg 1)$. The treatment is stopped by 
switching off the analog commutators, after the counter reaches a specified value, as proposed in Fig. 17. Another possibility could be to compare the state of the system at periodic times to check if it reaches stationarity. The final result is then loaded by the digital board via ADC (Analog-to-Digital Converter).

\section{Conclusion}

The authors have proposed a nonlinear electronic network inspired from transmission phenomena in biological systems. It allows the conception of circuits for high speed image processing based on front propagation principles. This article illustrates this on an active region algorithm, making a parallel between the point of view of morphological geodesic reconstruction and a-priori the more robust active curve approach, with the idea of using the different scales of regularity of the bistable propagation along the height of its front. Even if this efficient method is rather heavy, the article shows that an adapted physical implementation can overcome a technological break point. Therefore, it could generalize, for instance, the use of deformable templates. Further works will concern on the one hand the generalization of electronic front propagation manipulation (how to determine $B$ is the key point) in order to expand the field of applicable treatments and on the other hand the study of SOC (Systemon-Chip) possibilities, especially by adding FPGA based controller adapted to complex decompositions and allowing the completion of treatments by linear and probabilistic parts for example, mixing analog and numeric methods, in order to create a specific high-level programming and controlling system for "artificial retina"-type high speed image processing. High speed intelligent 
sensors development for the industry is one aim for these works.

\section{References}

[1] K. Kyuma, E. Lange, J. Otha, A. Hermanns, B. Banish and M. Ita, Artificial retinas - fast versatile image processors, Nature 372 (6502) (1994) 197-198.

[2] M. Barbaro, P.Y. Burgi, A. Mortara, P. Nussbaum and F. Heitger, A 100x100 pixel silicon retina for gradient extraction with steering filter capabilities and temporal output coding, IEEE J. Solid-State Circuits 37 (2) (2002) 160-172.

[3] O. Aubreton, B. Bellach, L.F.C. Yan Voon, B. Lamalle, P. Gorria and G. Cathbras, Retina for pattern matching in standart 0.6 $\mu \mathrm{m}$ CMOS technology, J. Electronic Imaging, SPIE 13 (3) (2004) 559-569.

[4] J. Serra, Image Analysis and Mathematical Morphology, Vol. I, London: Academic Press, 1982.

[5] J. Serra, Image Analysis and Mathematical Morphology, Vol. II : Theoretical Advances, London: Academic Press, 1988.

[6] S. Binczak, J.M. Bilbault and P. Marquié, Pattern dynamics in a nonlinear electrical lattice, Int. J. Bifurcation Chaos Appl. Sci. Eng. 13 (2003) 483-492.

[7] R. Goldenberg, R. Kimmel, E. Rivlin and M. Rudzsky, Fast Geodesic Active Contours, IEEE Trans. Image Proc. 10 (10) (2001) 1467-1475.

[8] N. Paragios and R. Deriche, Geodesic Active Contours and Level Sets for the Detection and Tracking of Moving Objects, IEEE Trans. Pattern Ana. and Machine Intel. 22 (3) (2000) 266-280.

[9] A. Zarandy, A. Stoffels, T. Roska and L.O. Chua, Implementation of binary and gray-scale mathematical morphology on the CNN universal machine, IEEE Trans Circuits and Systems I 45 (2) (1998) 163-168. 
[10] A.C. Scott, Active and nonlinear wave propagation in electronics, New York: Wiley-Interscience, 1970.

[11] L.O. Chua and L. Yang, Cellular Neural Network: Theory, IEEE Trans. Circuits Systems I, 35 (1988) 1257.

[12] A.C. Scott, Neuroscience: A Mathematical Primer, New York: Springer, 2002.

[13] A.C. Scott Edt., Encyclopedia of Nonlinear Science, New York: Routledge, 2005.

[14] J. Keener and J. Sneyd, Mathematical Physiology, Interdisciplinary applied mathematics, New York: Springer, 1998.

[15] S. Binczak and J.M. Bilbault, Experimental propagation failure in a nonlinear electrical lattice, Int. J. Bifurcation Chaos Appl. Sci. Eng. 14 (5) (2004) 18191830.

[16] J. Keener, Propagation and its failure in coupled systems of discrete excitable cells, ISIAM J. Appl. Math. 47 (3) (1987) 556-572.

[17] T. Erneux and G. Nicolis, Propagating waves in discrete bistable reactiondiffusion systems, Physica D 67 (3) (1993) 237-244.

[18] V.G. Fast and G. Kleber, Role of wavefront curvature in propagation of cardiac impulse, Cardiovascular research 33 (1997) 258-271.

[19] S. Jacquir, S. Binczak, P. Athias and J.M. Bilbault, A Theoretical Approach of the Propagation through Geometrical Constraints in Cardiac Tissue, Int. J. Bifurcation Chaos Appl. Sci. Eng., to appear in 2007.

[20] J.C. Neu, R.S. Preissig and W. Krassowska, Initiation of propagation in a onedimensional excitable medium, Physica D 102 (1997) 285-299.

[21] E. Sanchez-Sinencio and J. Silva-Martinez, CMOS transconductance amplifiers, architectures and active filters : A tutorial, IEE Proceedings Circuits, Devices and Systems 147 (1) (2000) 3-12. 
[22] B. Gilbert, A precise four-quadrant multiplier with subnanosecond response, IEEE J. Solid-State Circuits sc-3 (4) (1968) 365-373.

[23] J. Cosp and S. Binczak, Programmable VLSI Cubic-Like Function Implementation, Electronics Letters 42 (21) (2006) 1221-1222. 
Figure 1: A planar wavefront propagation using a greyscale representation where white corresponds to $v=1$ and black to $v=0$. The top left inset shows the marker. The other insets illustrate the propagating wavefronts at different times (in arbitrary units). Parameters: $a=0.1$ and $D=1$. Results obtained from numerical simulations ( $4^{\text {th }}$ order Runge-Kutta algorithm) of eq. (6).

Figure 2: $v_{100, n}$ at different times corresponding to the insets of Fig. 1, illustrating the constant velocity. The dotted line shows the marker, the continuous lines, the propagating wavefront at $t=40,80$ and 120 a.u.. Parameters : $a=0.1, D=1$. Results obtained from numerical simulations ( $4^{\text {th }}$ order Runge-Kutta algorithm) of eq. (6).

Figure 3: A circular wavefront propagation using a greyscale representation where white corresponds to $v=1$ and black to $v=0$. The top left inset shows the marker. The other insets illustrate the propagating wavefronts at different times (in arbitrary units). Parameters: $a=0.1$ and $D=1$. Results obtained from numerical simulations ( $4^{\text {th }}$ order Runge-Kutta algorithm) of eq. (2).

Figure 4: Propagation velocity versus the diffusive parameter $D$. Numerical results comparison between planar wave speed (continuous line) and circular wave speed (dotted line). Parameter : $a=0.1$. 
Figure 5: Propagation of a wavefront in an inhomogeneous grid. Inset (a) shows the initial marker and an object $\Theta$. Inset (b) shows the wavefront crossing the corridor (of width $\mathrm{W}$ and thickness $\mathrm{T}$ ) separating the two parts of $\Theta$ at $t=100$ a.u.. Inset (c) shows the propagation of a circular wave emerging from the corridor at $t=200$ a.u.. Inset (d) shows the final stationary state, obtained at $t=300$ a.u. Parameters: $a=0.1, D=1$, $W=6$ nodes, $T=4$ nodes.

Figure 6: Unsuccessful propagating wave via a corridor $\mathrm{a}=0.1, \mathrm{~W}=6$ nodes, $\mathrm{T}=4, \mathrm{D}=1.2$

Figure 7: Diffusive parameter $D$ versus width $W$ curves for different values of thickness $T$ of $\Theta$. When $D$ is above this curve the wavefront is pinned. When $D$ is beneath this curve, the wavefront can cross the corridor and propagate. Parameter: $a=0.1$

Figure 8: Determination of the propagation conditions depending on the size of the marker the diffusive parameter $D$. The border above (continuous line) corresponds to a collapse of the marker due to a too strong diffusive parameter. The border beneath (dotted line) corresponds to the propagation failure due to too weak coupling. Parameter: $a=0.1$. Initial marker: square whose width ranges between 1 and 10 nodes.

Figure 9: Sifter effect (Top: shape, middle: marker, bottom: propagation blockage) for different values of $c$ : From left to right: $c=0.25$ to $c=4.75$ by incremental steps of 0.75 . 
Figure 10: Isotropy (Top: shape, middle: marker, bottom: propagation break) in the case where $c=2$.

Figure 11: Scale aspect of c: Porosity effect (up: shape and marker, down: propagation blockage for increasing values of $c$ from left to right and from top to bottom: $c=0.1$ to $c=1.75$ by incremental steps of 0.15$)$.

Figure 12: A simple example of edge restoration/interpolation with an interior marker in the case where $c=3$. Left: starting configuration, Right: final configuration.

Figure 13: Scale aspect with high c: Hull effect (with interior and exterior markers). Left: Final state. Right: Shape and markers. Parameter: $c=8$. For this example, the marker rule is not set.

Figure 14: Secondary geometrical scale aspect of h. From left to right, then top to bottom, $\mathrm{h}$ increases in a ratio-2 geometrical manner from $h=0.0078$ to $h=0.996 . c=8$.

Figure 15: Multiscale segmentation in one-pass in the case where $c=8$. The top insets show the marker and the starting configuration (right) composed of four parts separated by 11 nodes vertically and 15 nodes horizontally and the final state (left). The resulting fuzzy segmentation is obtained, from left to right then top to bottom, for $h=0.35, h=0.5, h=0.7$ and $h=0.996$.

Figure 16: Grey level segmentation of the noisy regions "background between columns" and "right cheek", starts with small markers. From left to right 
and top to bottom : Original image; binary results showing the detection of the background and the right cheek; these two regions isolated in the original image; distribution of the markers (one of the two markers can be seen in the background) and of the diffusive parameter $D_{n, m}$ calculated with eq. $(15)$.

Figure 17: Scheme of the mixed-signal architecture.

Figure 18: Analog network.

Figure 19: Detail of the analog network. 

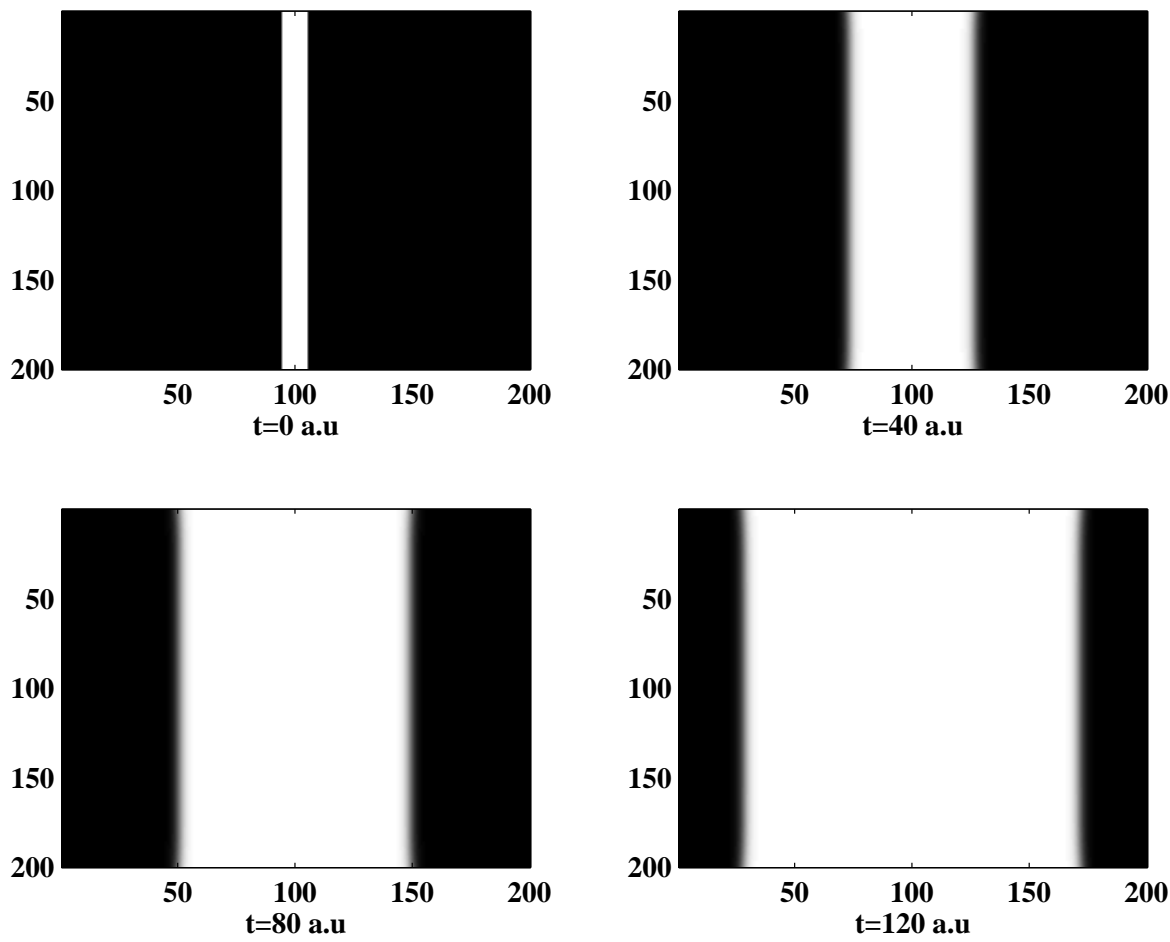

Fig. 1. 


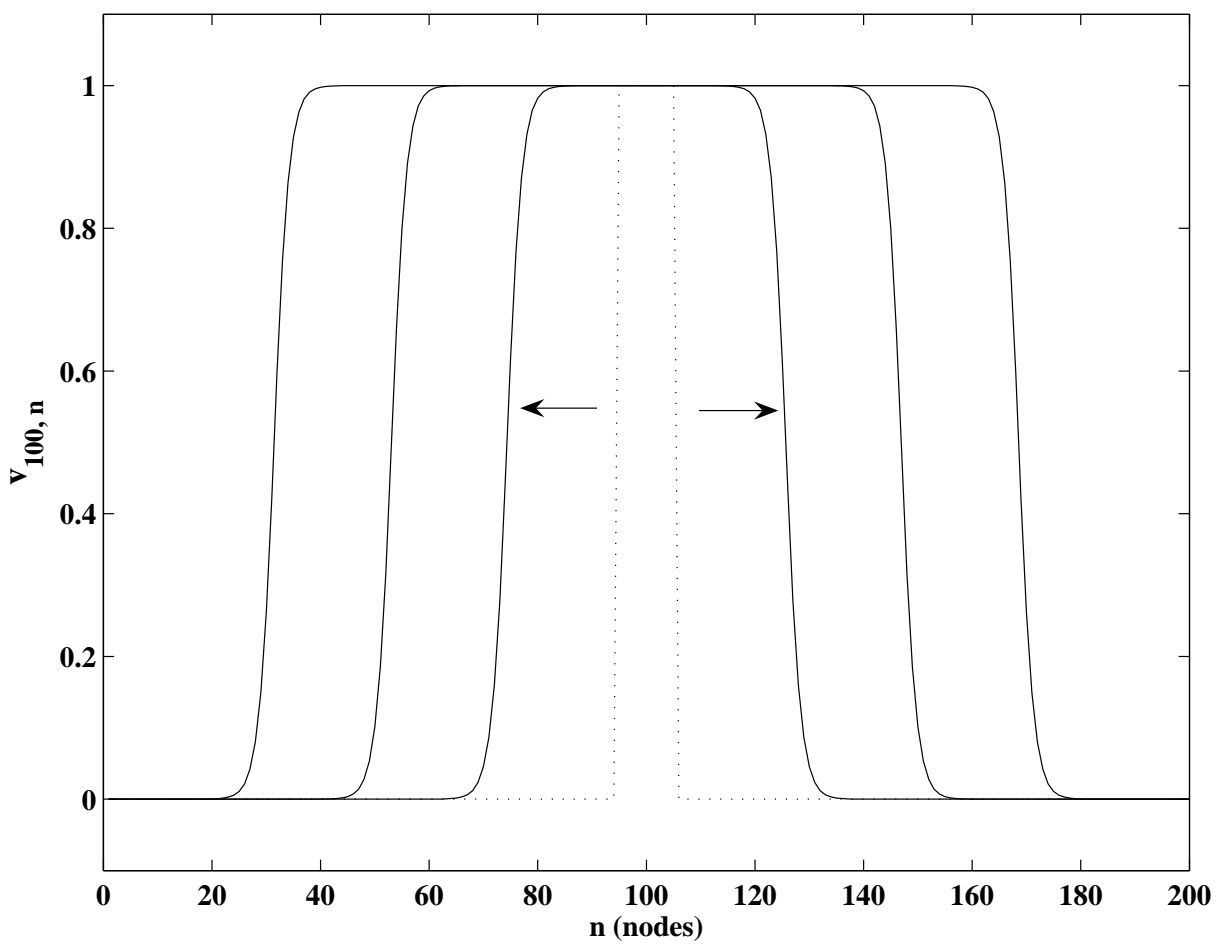

Fig. 2. 

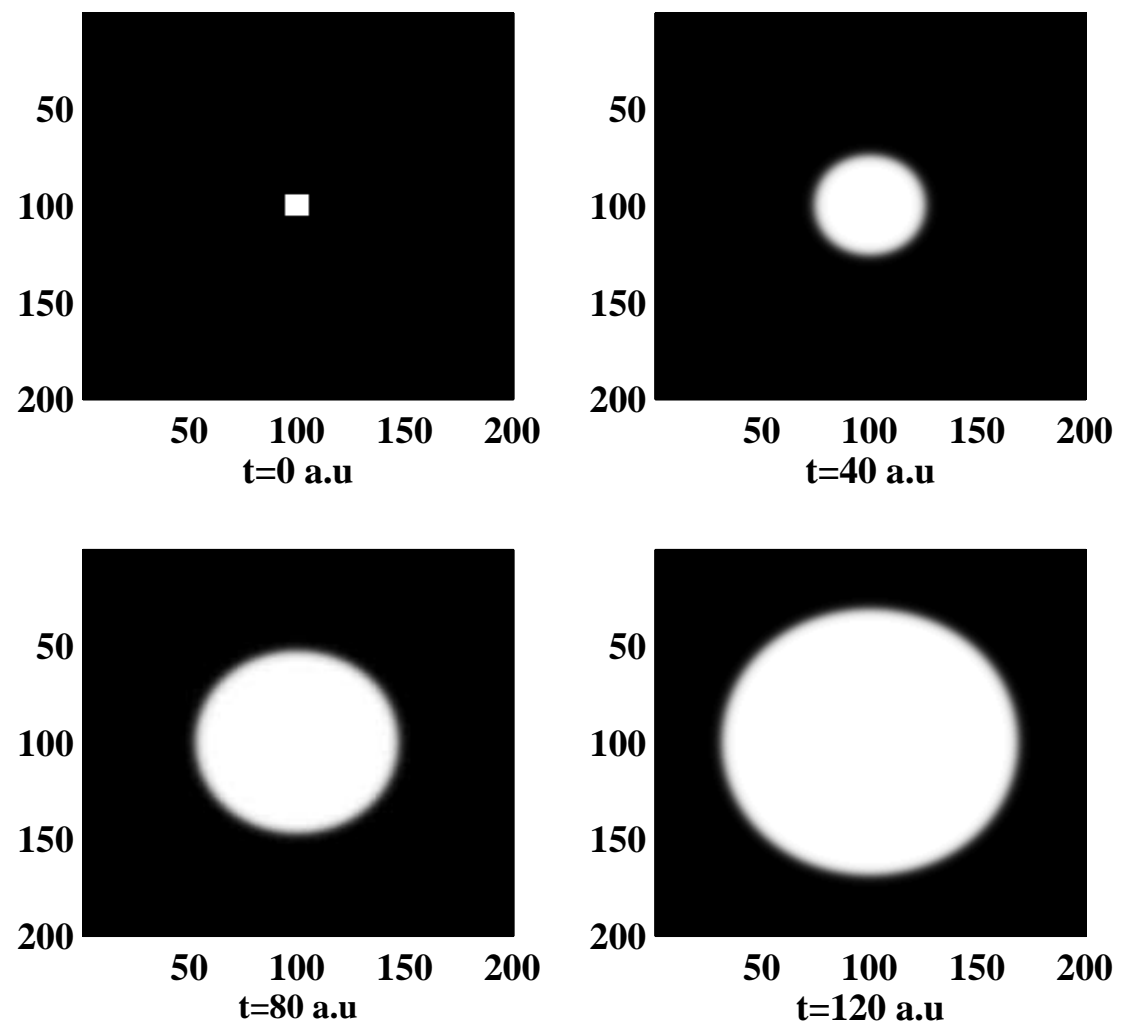

Fig. 3. 


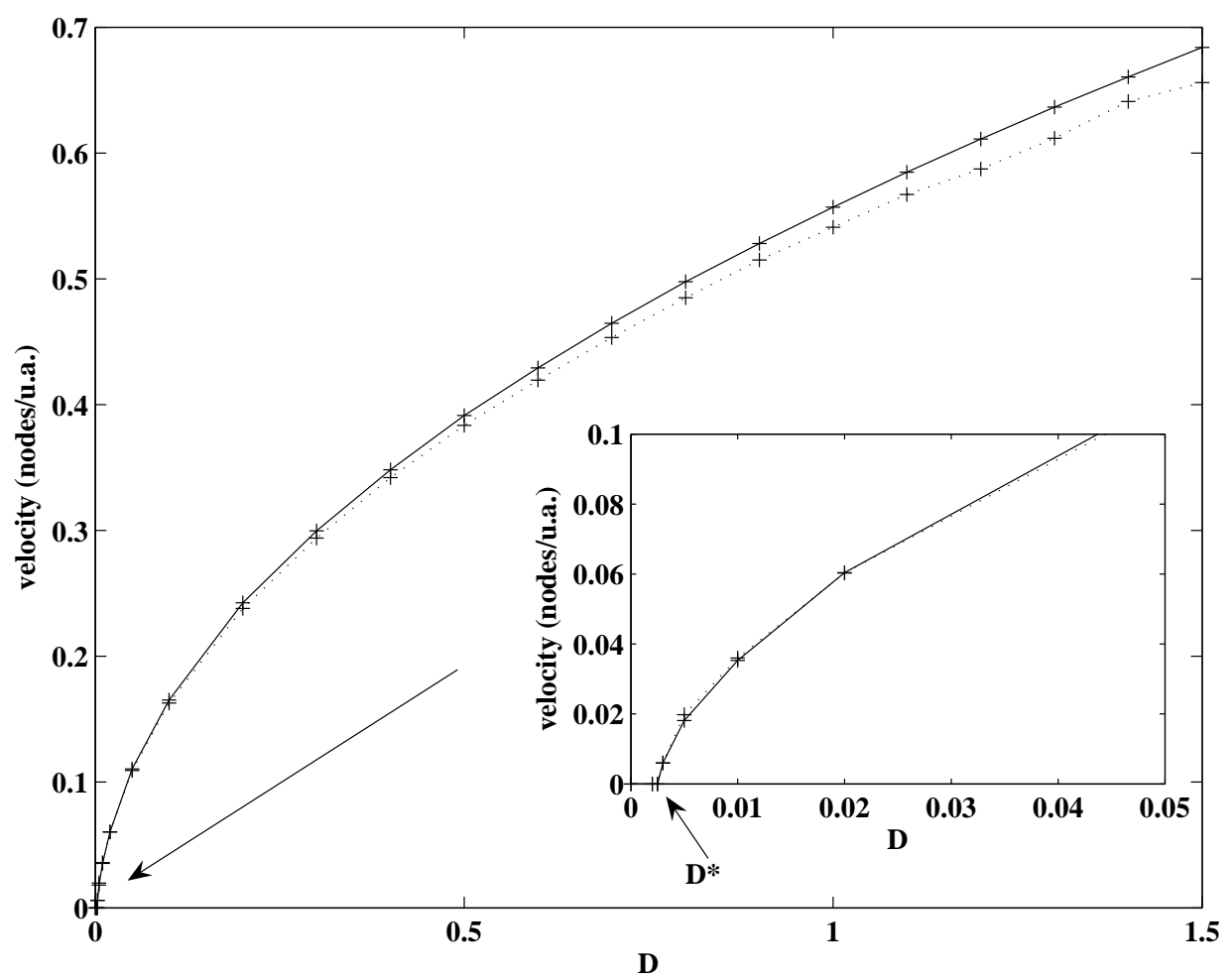

Fig. 4. 

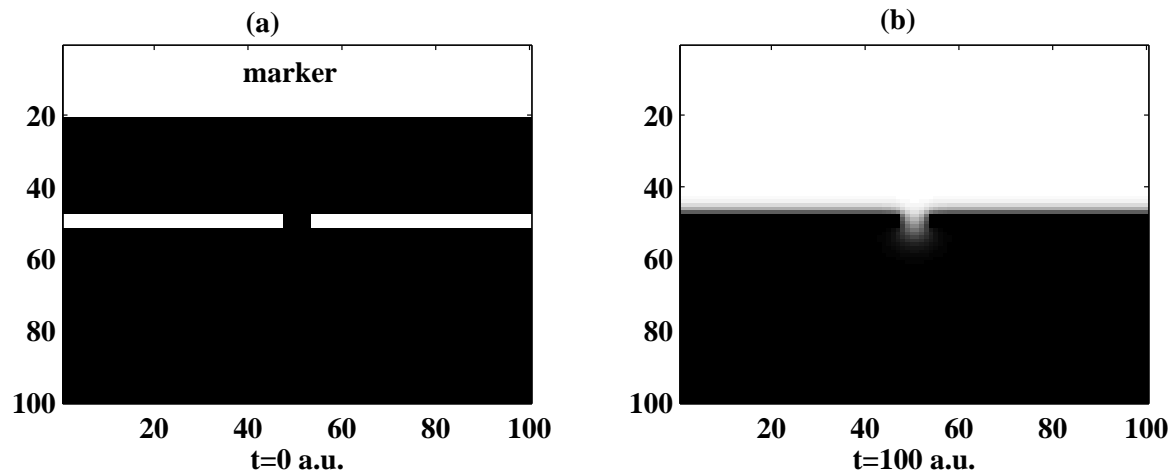

(c)
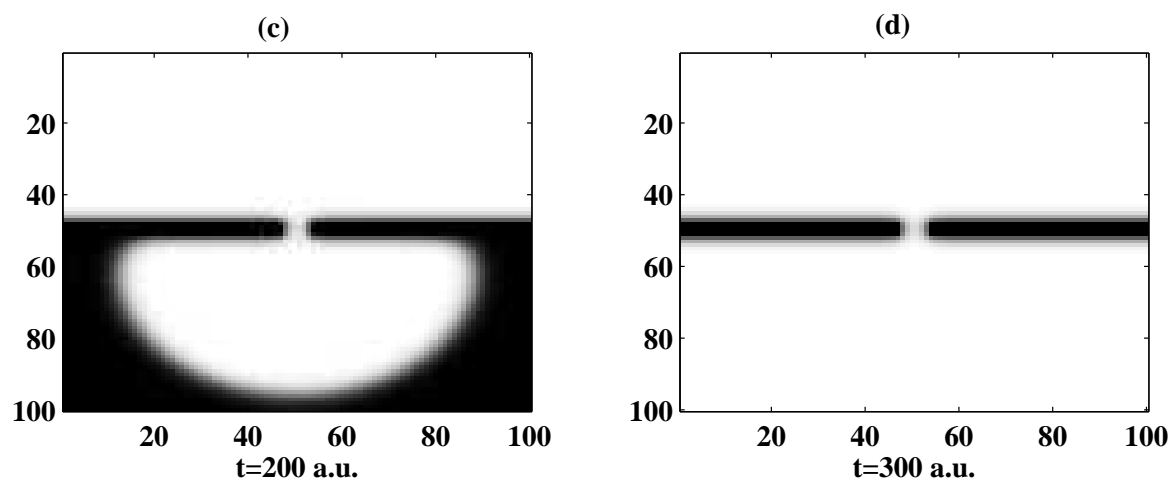

Fig. 5 . 


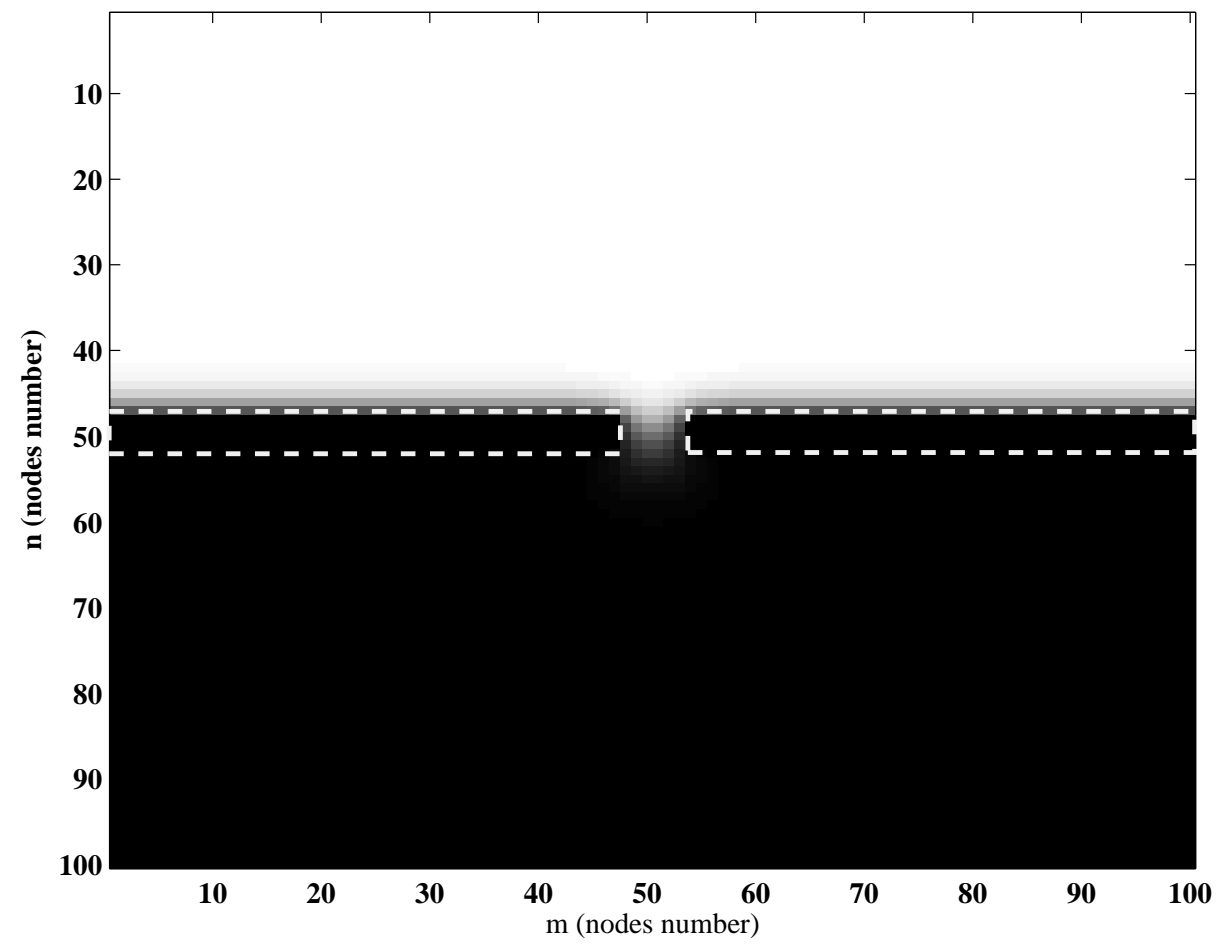

Fig. 6 . 


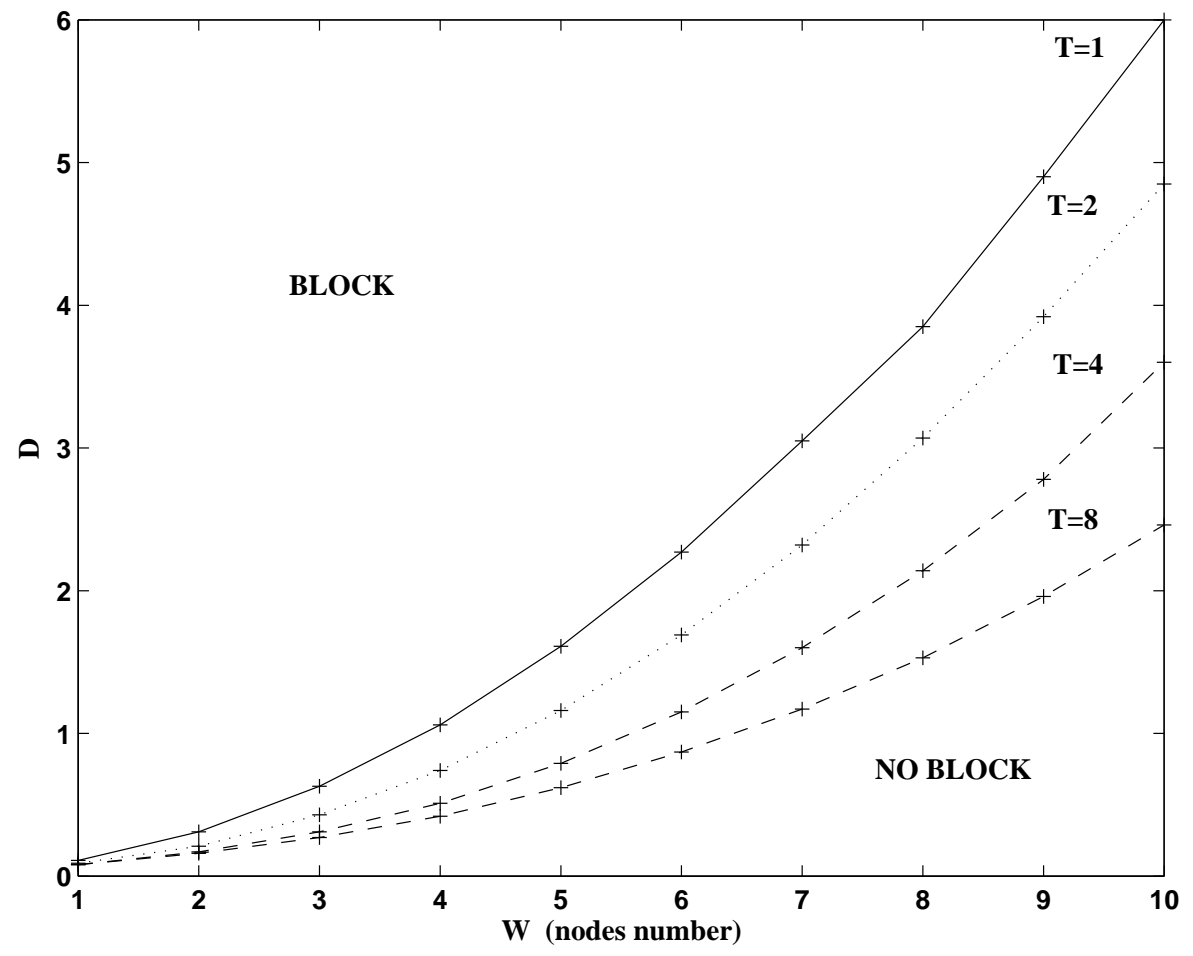

Fig. 7. 


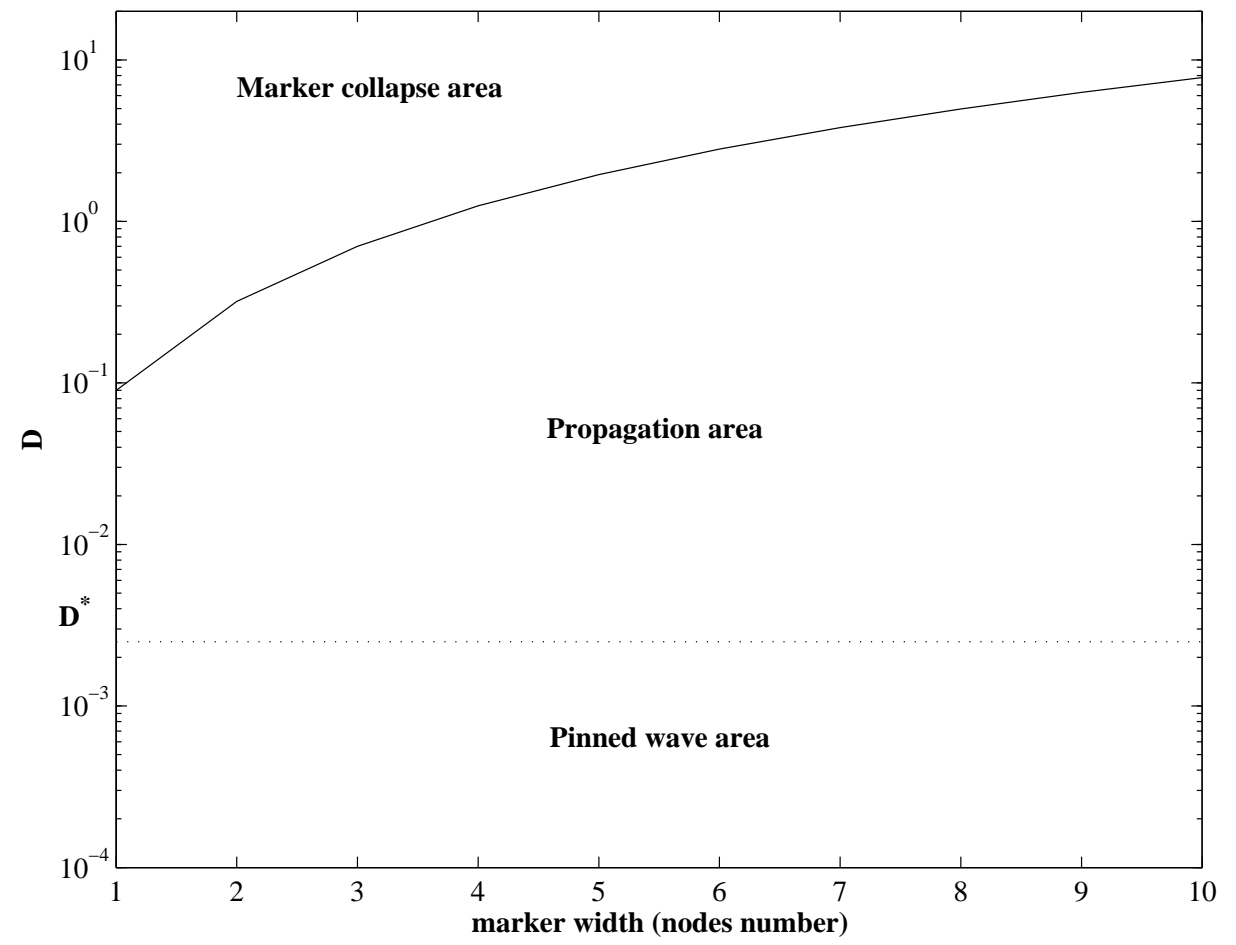

Fig. 8 . 


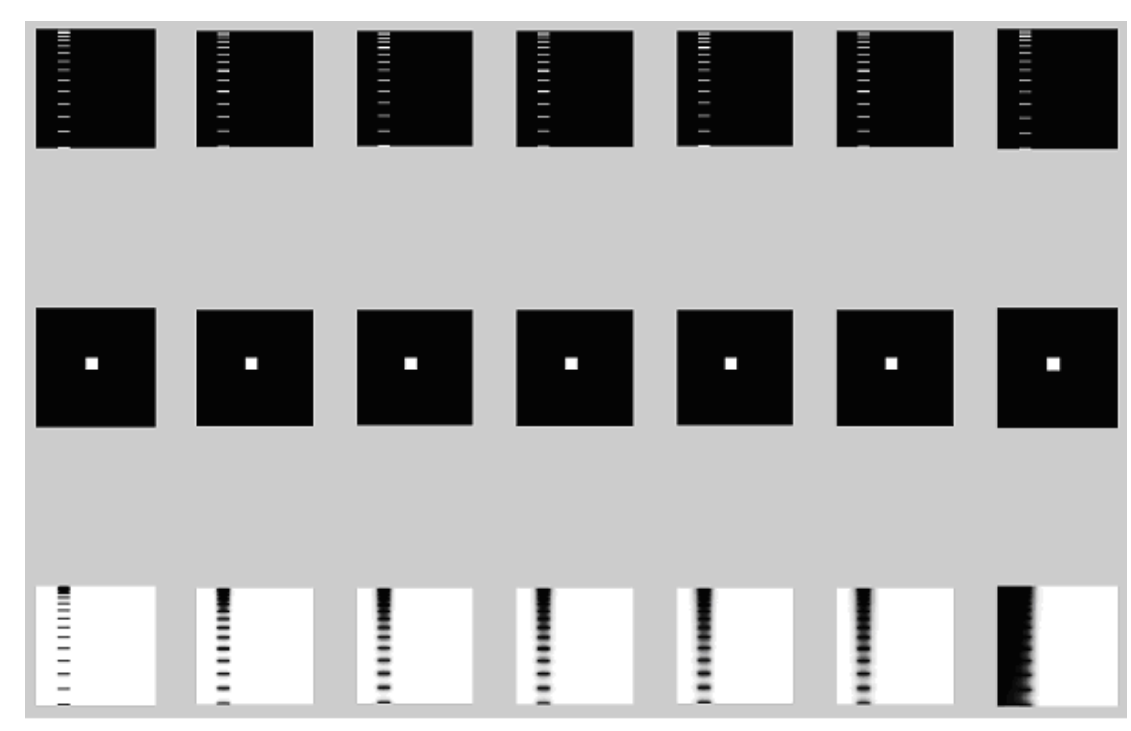

Fig. 9. 


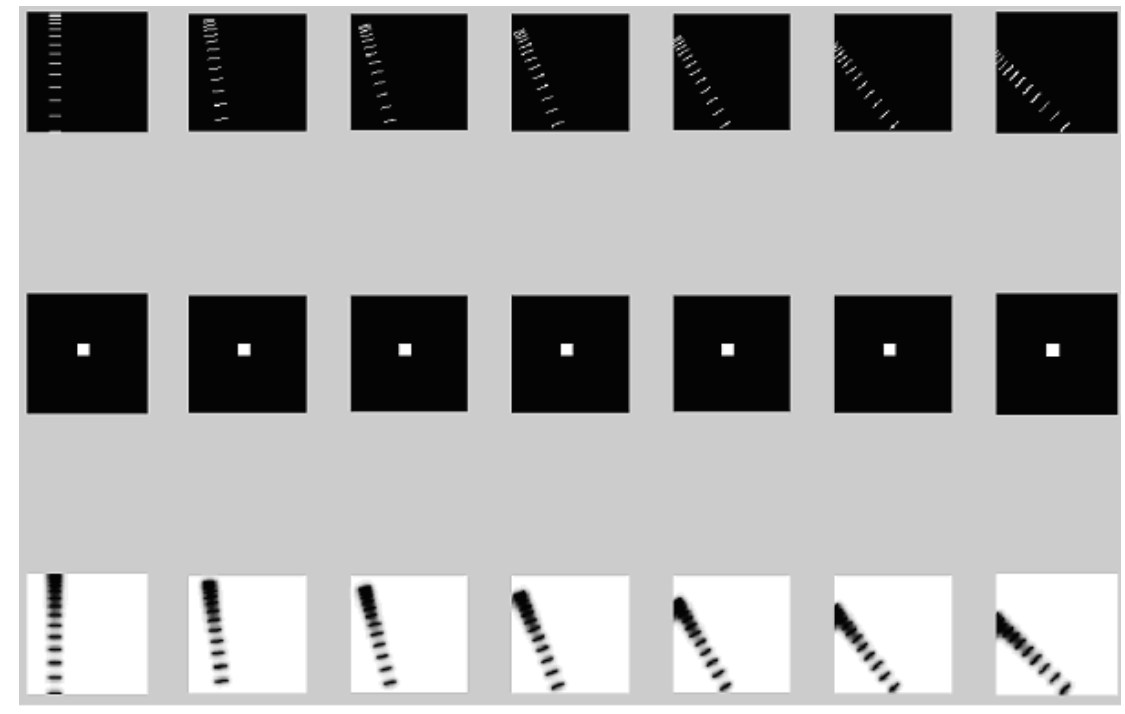

Fig. 10. 

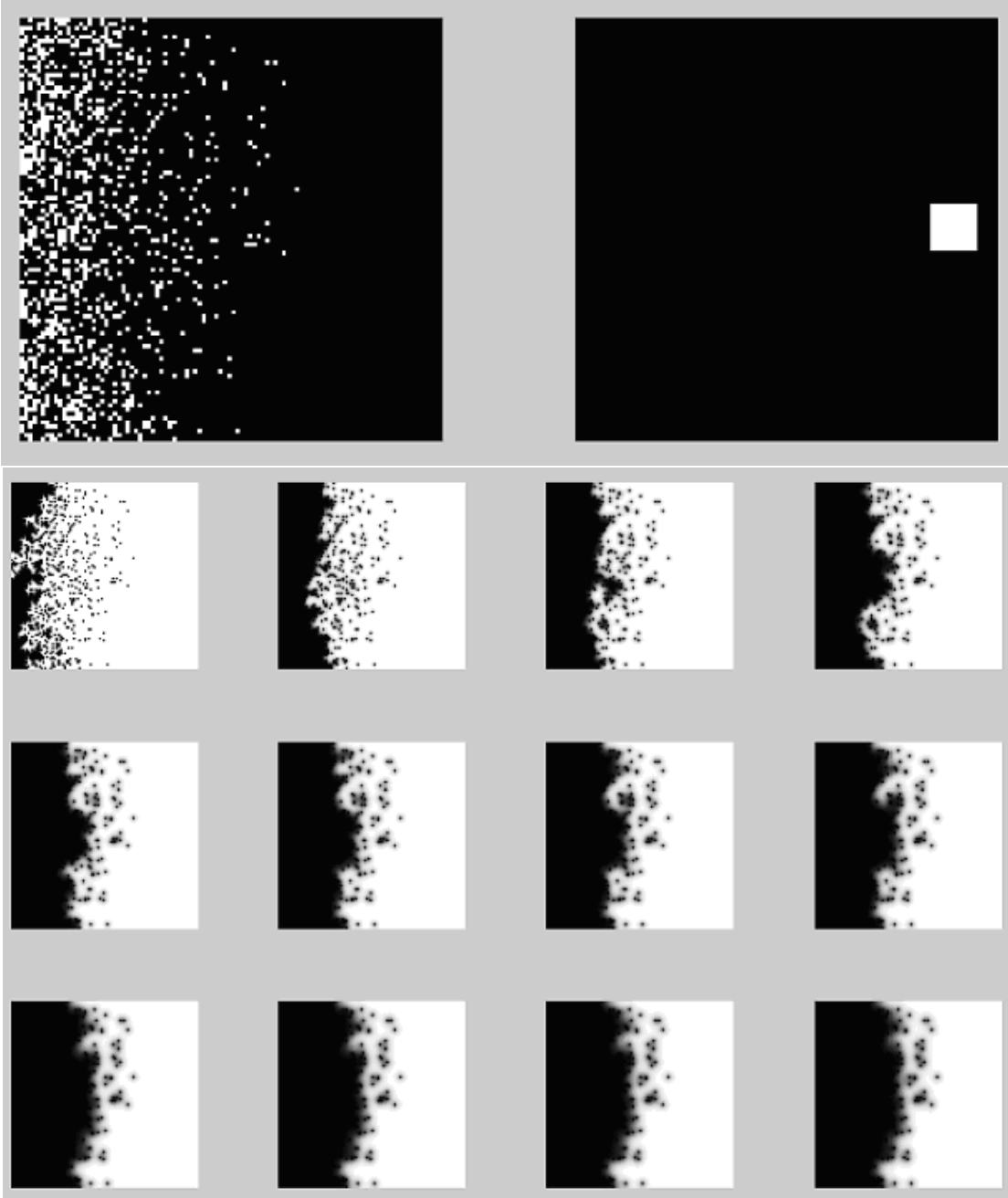

Fig. 11. 

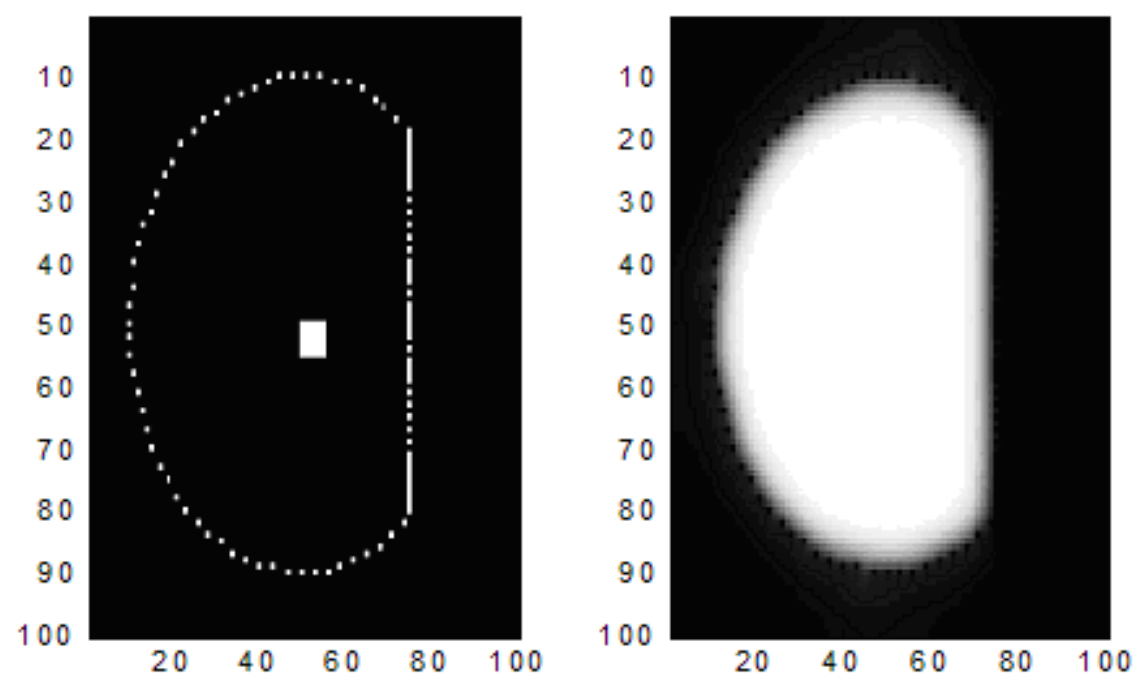

Fig. 12 . 


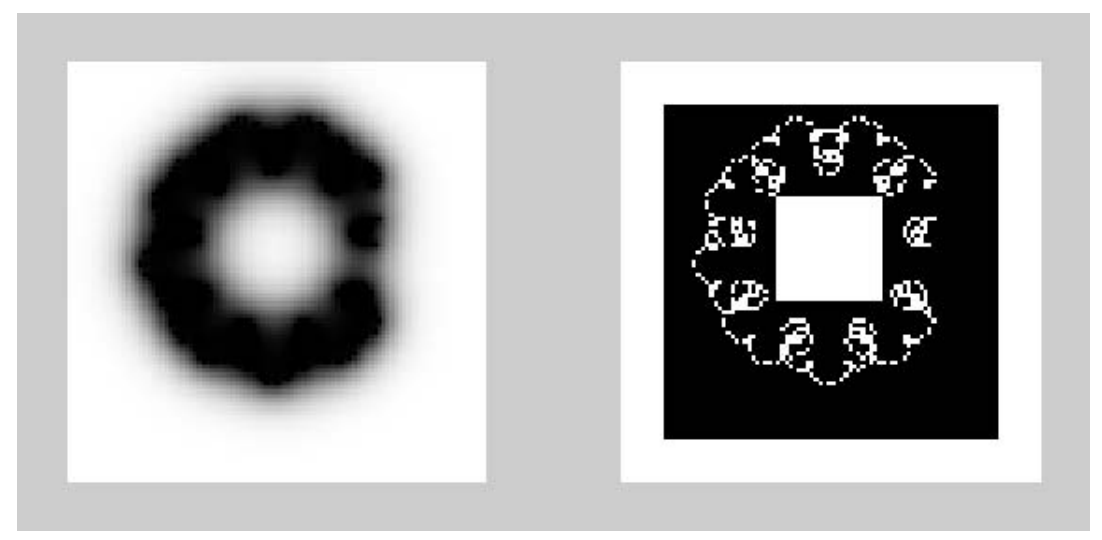

Fig. 13. 


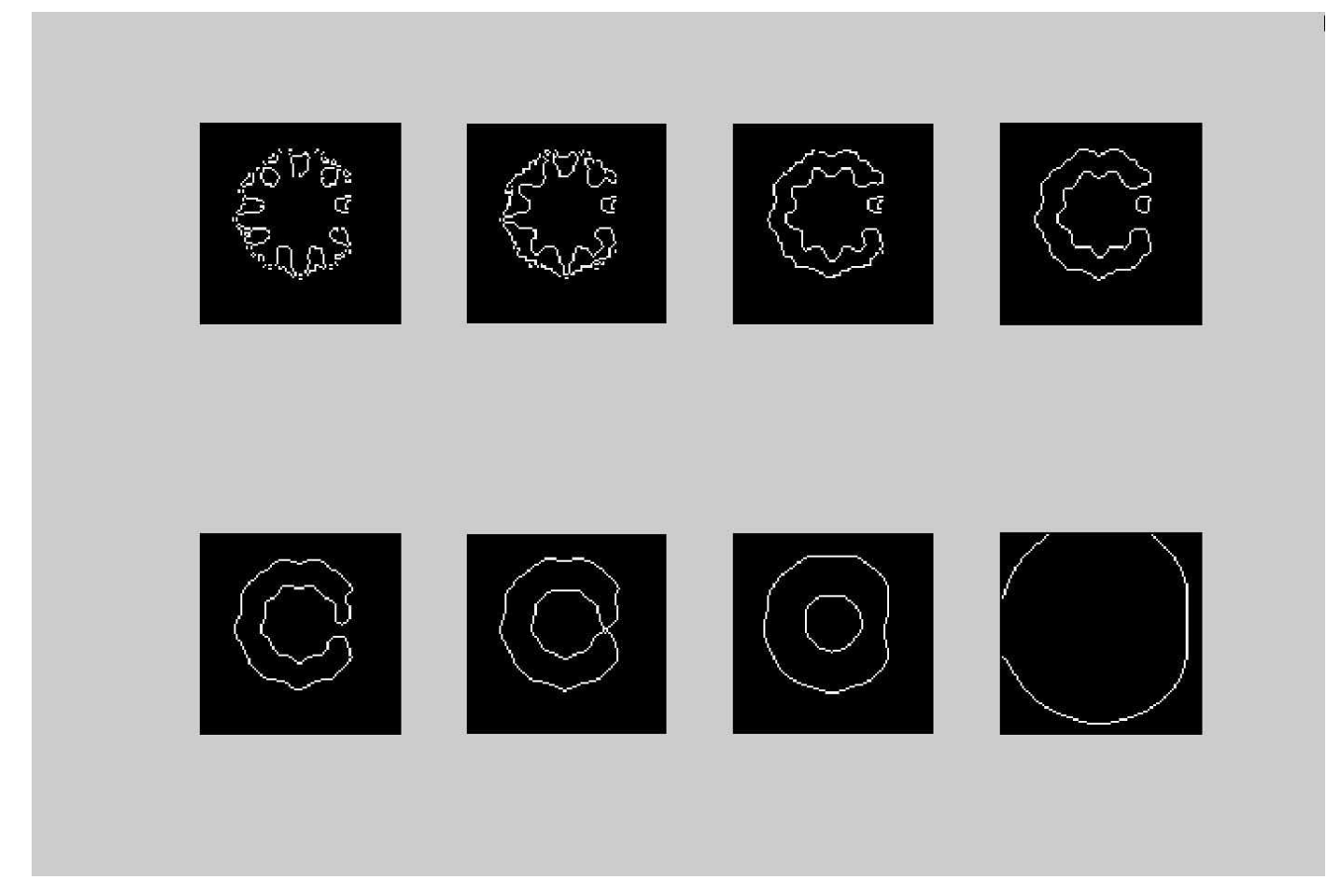

Fig. 14. 


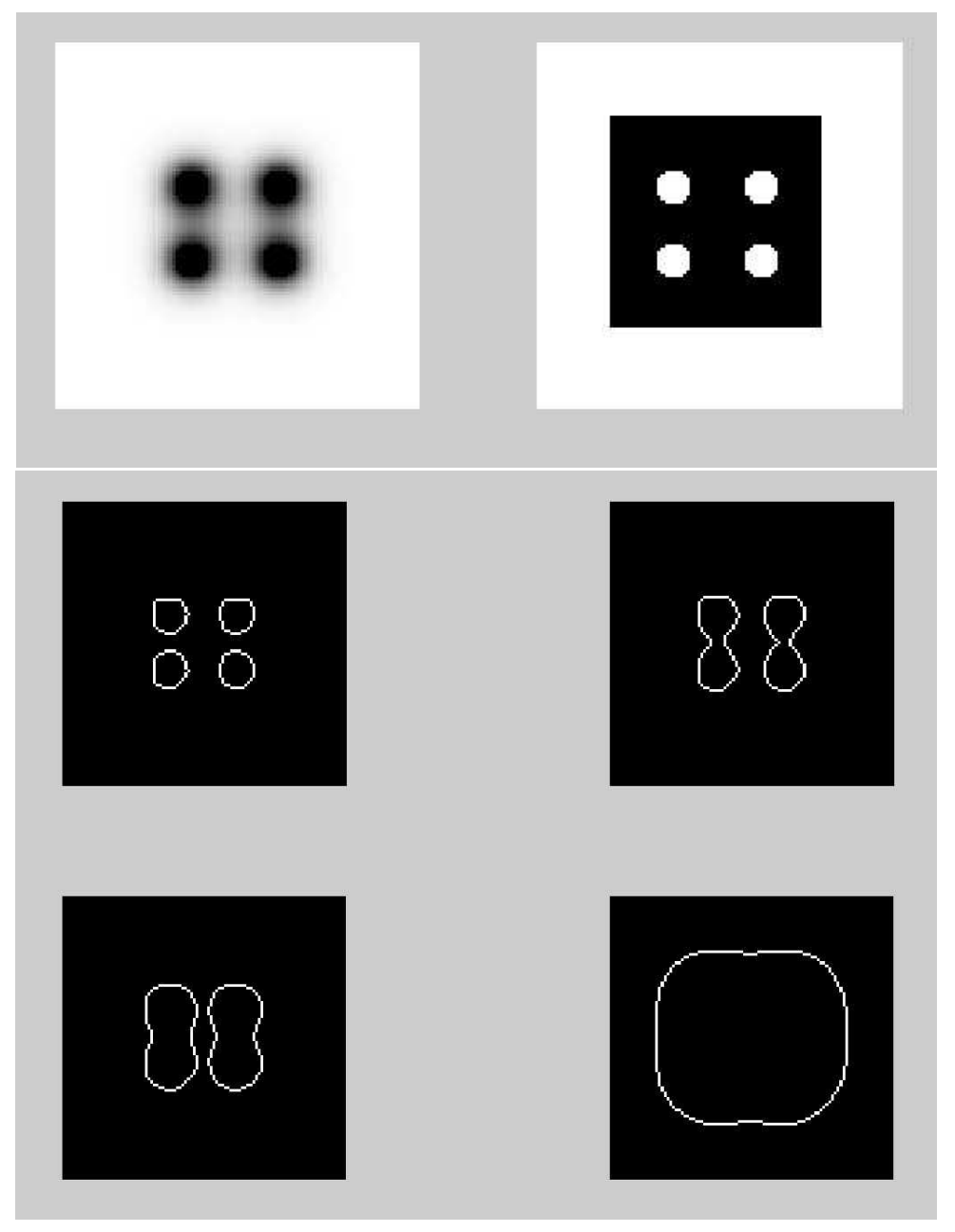

Fig. 15. 

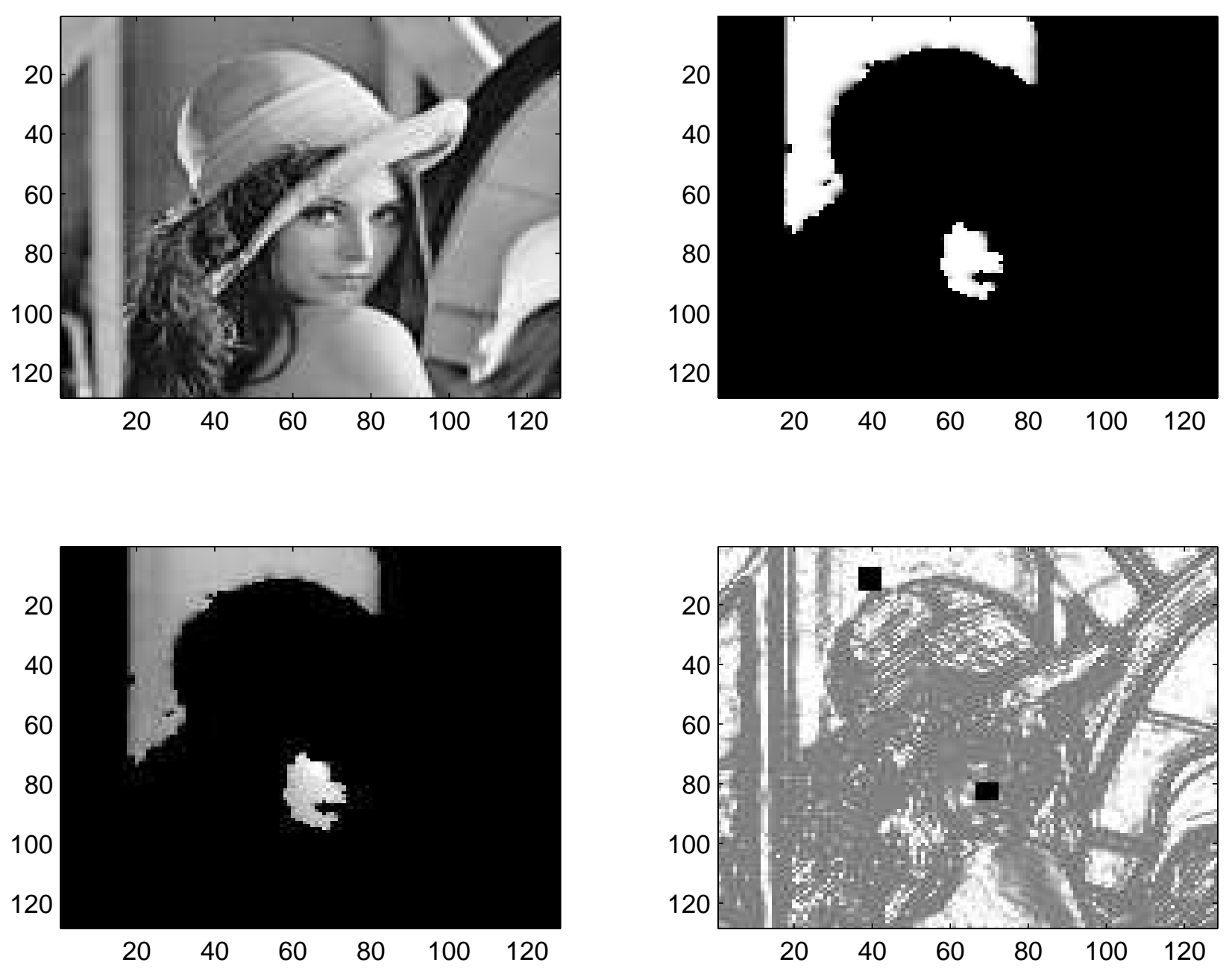

Fig. 16 . 


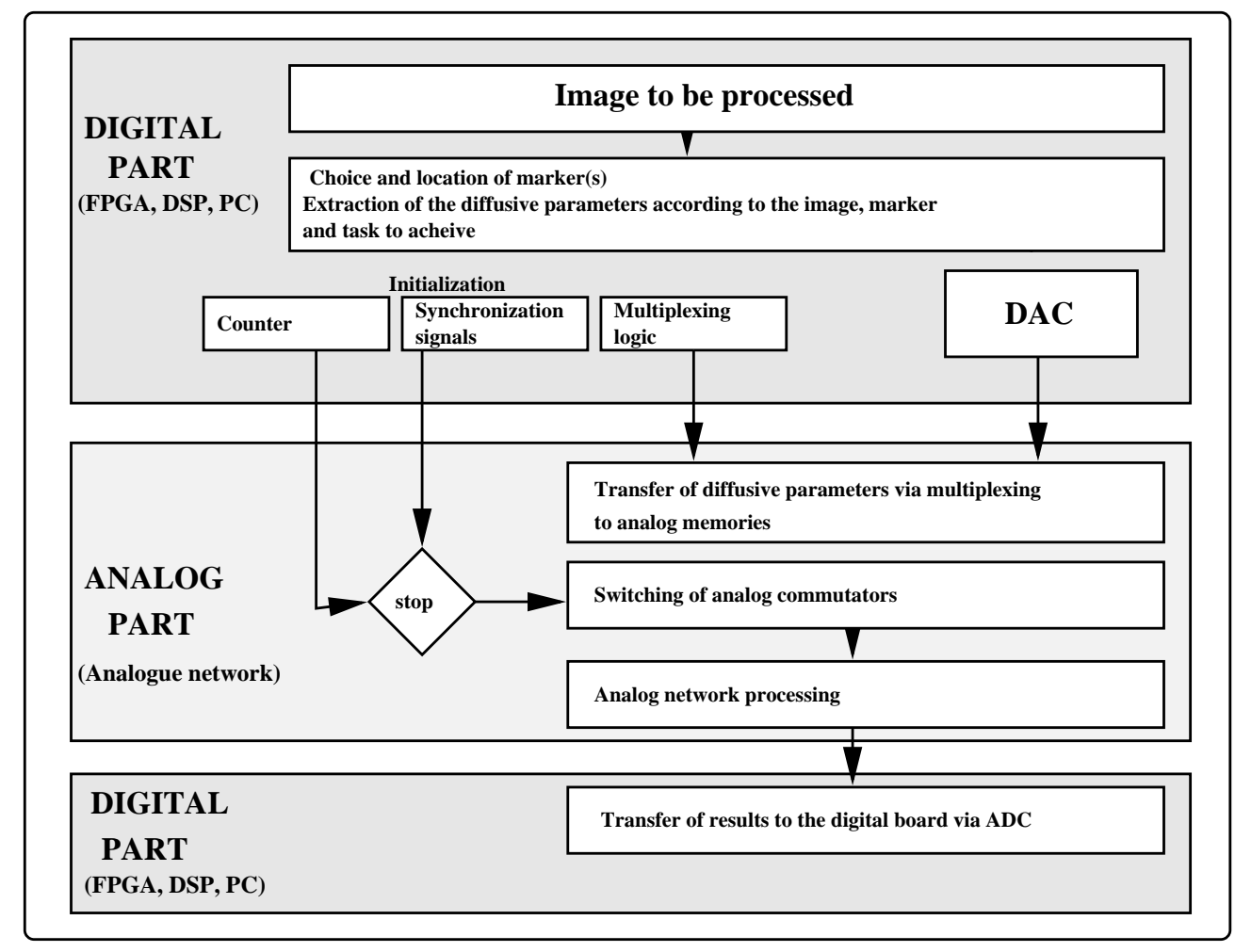

Fig. 17. 


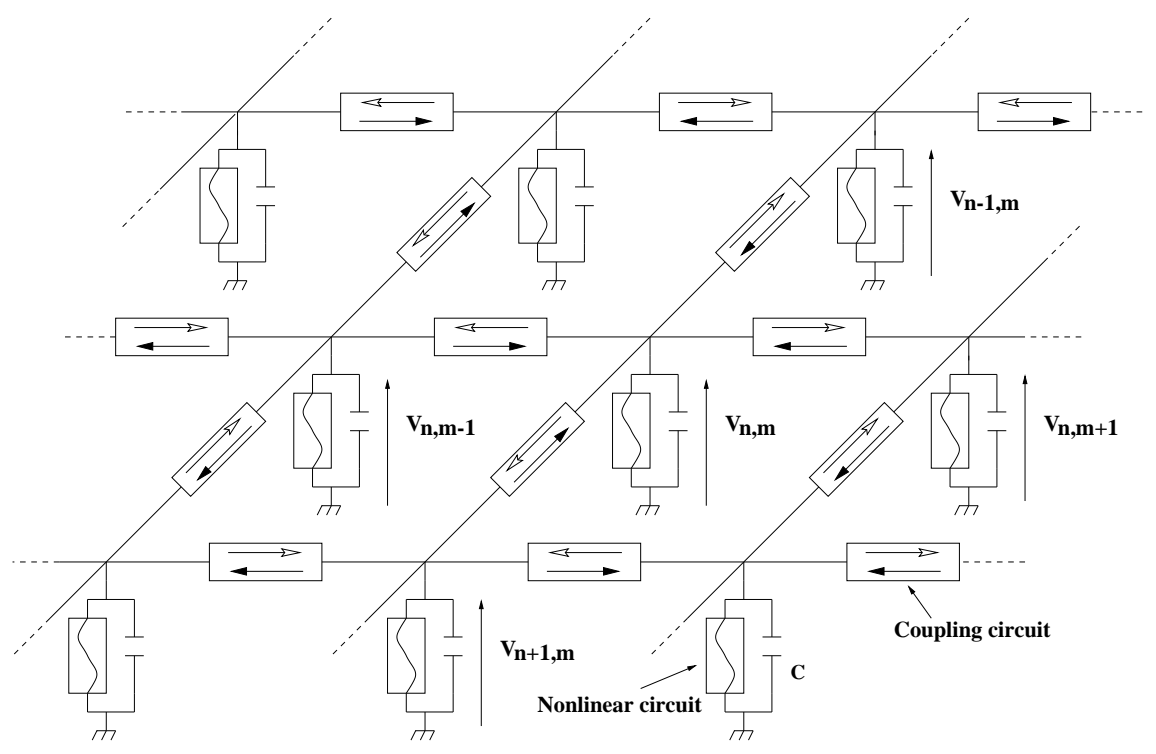

Fig. 18. 


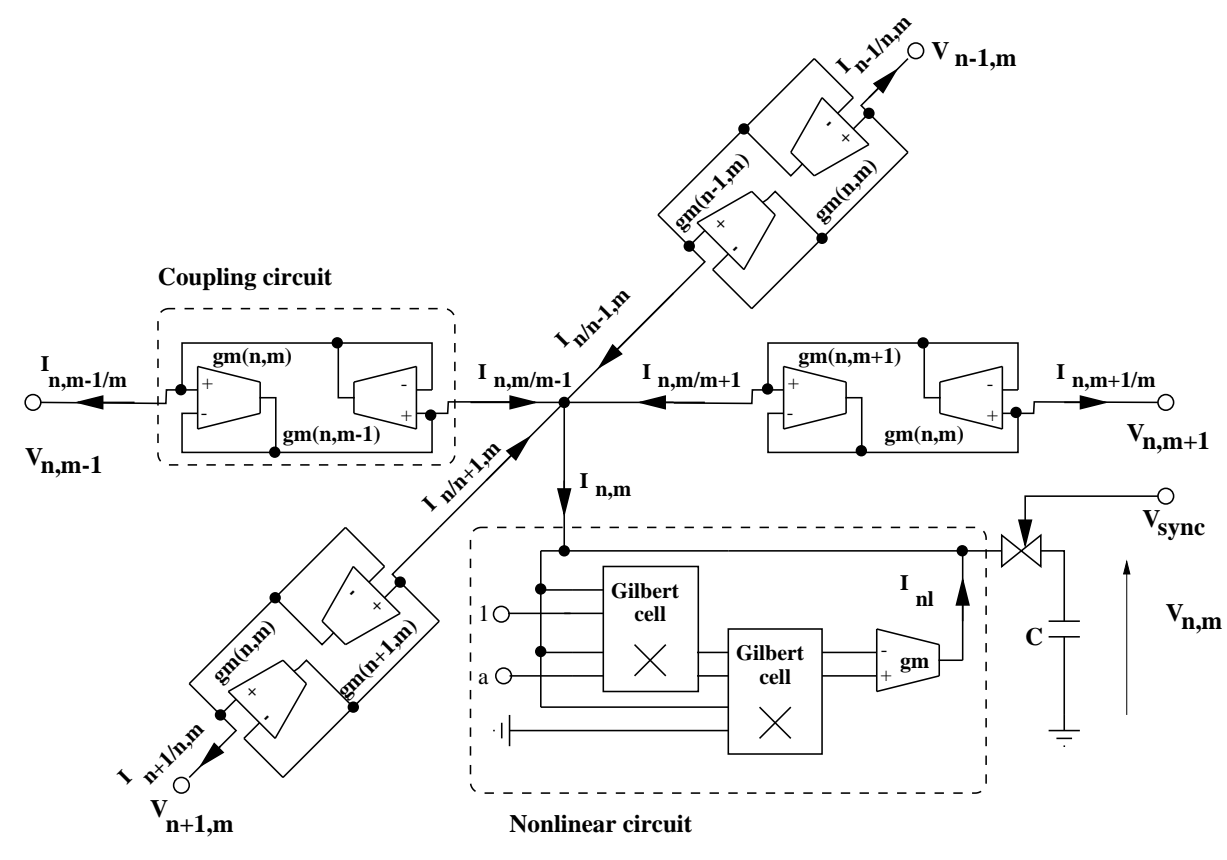

Fig. 19. 